\title{
Detecting Deception via Eyeblink Frequency Modulation
}

To assess the efficacy of using eyeblink frequency modulation to detect deception about a third party, 32 participants were sent on a mission to deliver a package to an interviewer. 17 of the participants lied to the interviewer about the details of their mock mission and 15 responded truthfully. During the interview, eyeblink frequency data were collected via electromyography and recorded video. Liars displayed eyeblink frequency suppression while lying, while truth tellers exhibited an increase in eyeblink frequency during the mission relevant questioning period. The compensatory flurry of eyeblinks following deception observed in previous studies was absent in the present study. A discriminant function using eyeblink suppression to predict lying correctly classified $81.3 \%$ of cases, with a sensitivity of $88.2 \%$ and a specificity of $73.3 \%$. This technique, yielding a reasonable sensitivity, shows promise for future testing as, unlike polygraph, it is compatible with distance technology. 
1

2

3

4

5

7

8

9

10

11

12

13

14

15

16

17

18

19

20

21

22

23

24

25

26

27

28

29

30

31

32

33

34

35

36

37

38

39

40

41

42

43

44

45

46

47
Brandon S. Perelman

Michigan Technological University

Department of Cognitive and Learning Sciences

Houghton, MI

\section{Corresponding Author: Brandon S. Perelman \\ Michigan Technological University 1400 Townsend Drive \\ Department of Cognitive and Learning Sciences}

Harold Meese Center, Office 203

Houghton, MI 49931

(610) $331-4341$

BPerelman@gm.slc.edu 
Detecting Deception via Eyeblink Frequency Modulation

\section{Introduction}

Modern deception detection methods testing physiological indices of deception use

techniques such as galvanic skin response (GSR; e.g., Carmel et al., 2003), fMRI (e.g. Bhatt et al., 2009; Langleben, 2005; Shah et al., 2001), and EEG detected P300 event related potentials (Abootalebi, Moradi, \& Khalilzadeh, 2006; Ambach et al., 2010; Meijer et al., 2007). In the academic literature, responses are sometimes elicited (e.g., Langleben, 2008) using some variation of the Guilty Knowledge Test (GKT; Lykken, 1957; 1959). Though these physiological techniques may provide strong sensitivity and specificity in laboratory settings (MacLaren, 2001), they require proprietary equipment, proximity to the suspect, and suspect awareness of the analysis. Furthermore, classification accuracy of GKT based tests obtained in laboratory settings from mock crime scenarios may not generalize to naturalistic settings (Carmel et al., 2003). The present study explores a known behavioral indicator of deception, cognitive demand modulated blink frequency $(\mathrm{BF})$. BF modulation is an attractive behavioral indicator of deception because BF data may be collected using hidden cameras or distance technology (i.e., web cams) and analyzed surreptitiously either in real time or post hoc from recorded video. Furthermore, BF data collection does not require any special equipment or questioning schemes. BF modulation has been experimentally validated using both GKT based questioning methods reliant upon recognition (Leal \& Vrij, 2010) and a conversationally natural free recall method (Leal \& Vrij, 2008). The present study builds upon previous research using a mission based scenario in which the deception pertains to a third party, under conditions that more closely resemble an interaction using distance technology. In particular, the present study accounts for variance attributable to cognitive demand resulting from participants monitoring the interviewer's body language. As the literature regarding deception about a third party is somewhat sparse, the study expands prior 
78 work on third party deception to include validation using BF based measures of cognitive

79 demand.

\section{Cognitive Demand Modulated BF and Deception Detection}

The cognitive demand hypothesis follows that deception is more demanding than truth

82 telling because, in addition to recall and speech production required during truth telling,

83 deception requires suppressing deceptive cues in body language (DePaulo, 1988; Ekman, 1989),

84 fabricating an alternate story, and carefully monitoring that story to ensure that it does not

85 contradict interviewer knowledge of the event (Leal \& Vrij, 2008). Cognitive demand during

deception is detectable in experimental settings using measures such as response time (Gronau,

Ben-Shakhar, \& Cohen, 2005; Seymour, Kerlin, \& Kurtz, 2003) and startle response modulation

(Cacioppo, 2006; Verschuere et al., 2007). Cognitive demand during deception is also observed in real high stakes police interviews (Mann, Vrij, \& Bull, 2002).

$\mathrm{BF}$ based deception detection techniques use cognitive demand induced BF suppression to

91 indicate deception. Numerous studies demonstrate that increases in cognitive demand cause a

92 reduction in BF (e.g. Drew, 1951; Siegle, Ichikawa, \& Steinhauser, 2008). In addition to BF

93 suppression during lying, liars may also display a flurry of compensatory blinks after lying (Leal

$94 \&$ Vrij, 2008). Importantly, truth tellers in that experiment exhibited an increase in BF during the

95 relevant questioning period. While $\mathrm{BF}$ is correlated with many physiological and emotional

96 states, this effect may be partially explained by the accusatory subject matter of the questions

97 delivered during that period.

\section{Detecting Deception about a Third Party}

99 The majority of deception research has focused on subjective deception, or deception

100 related to a personal transgression (Iacono, 2000). However, recent work has broadened the

101 literature to include lying about characteristics of a third party (Bhatt et al., 2009; Leal et al., 102 2011; Meijer et al., 2007; Shah et al., 2001). Deception regarding a third party may differ 
103 characteristically from subjective deception because the stakes may be perceived as lower.

104 Response times (Haque \& Conway, 2001) indicate that recalling autobiographical information is

105 more demanding than semantic recall, however the literature is unclear regarding differences in

106 cognitive demand during deception about these types of information. Establishing these

107 characteristics is important, since detecting deception about person recognition or familiarity may

108 provide a means for establishing group affiliation, which in particular is not reliably detectable

109 via traditional polygraph tests (Sullivan, 2007).

110 While comparative cognitive demands associated with discussing these topics truthfully

111 and deceptively are not well understood, a number of studies explore deception about a third

112 party using other means. In a novel experiment, Leal et al. (2011) asked participants to participate

113 in mock espionage mission similar to the mock crime scenarios used by Lykken $(1957 ; 1959)$ to

114 validate the GKT. Participants were briefed by one of the experimenters who revealed personal

115 characteristics (i.e., hobbies), and later they were asked to identify and describe this experimenter

116 from amongst a set of photographs. Cognitive demand as rated by observers, as well as

117 differences in gaze direction, allowed discrimination between liars and truth tellers.

\section{Hypotheses}

119 First, differences in BF between liars and truth tellers should be similar to those described

120 in cases of subjective deception by Leal and Vrij (2008). Second, liars are expected to exhibit a

121 reduction in BF while lying, followed by a compensatory flurry of blinks. Finally, truth tellers are 122 expected to exhibit an increase in BF while answering the mission relevant questions.

\section{Method}

\section{Participants}

125 The Saint Joseph's University (Philadelphia, PA) IRB board approved (IRB 2012-12) 34

126 participants (25 female, 9 male, age 18-21) for the experiment from the undergraduate population 127 enrolled in introductory level psychology classes. All participants signed informed consent forms 
128 and were informed of the physiological measures, though they were blind with regard to the

129 specific aspects of the electrooculography (EOG) data used in the analysis. One participant was

130 excluded due to a failure to adequately follow the directions of the experiment and another

131 participant exhibited an exceptionally low BF $\left(D_{i}=2.16\right)$. The sample used in the analyses

132 therefore consisted of 23 females and nine males after exclusions.

\section{Data Collection and Analysis}

134 Eyeblinks were monitored using an Apple iSight camera mounted on a modified Yukon

135 Advanced Optics Inc. night vision head mount kit and positioned roughly 2 inches from the eye.

136 For convenient analysis, blink frequencies were also recorded via EOG using AD Instruments'

137 PowerLab 26T and the LabChart Pro v. 7 software package. Three electrodes, one on the

138 orbicularis oculi muscle, one on the frontalis muscle, and a third on the ear as ground monitored

139 eyeblinks in accordance with the protocol outlined by Conduit (2012) for monitoring blink

140 amplitude. Since the present study is concerned only with quantifying blink frequency, no

141 electrodes were placed to monitor more subtle eye movements. An Apple Macintosh iMAC

142 computer was used for collecting and analyzing data. Blink data was recorded continuously, and

143 quantification began immediately after the interviewer read the question and continued until the

144 participant's response terminated. Blinks were quantified manually from concurrent recorded

145 video of the eye from which EOG data was recorded. During this quantification, the experimenter

146 was blind to the participant's experimental condition. The EOG data were amplified (using the

147 default sampling rate of $1 \mathrm{KHz})$ and filtered (Range $=2 \mathrm{mV}$, Low Pass $=10 \mathrm{~Hz}$, High Pass $=.5$

$148 \mathrm{~Hz}$ ) and normalized in terms of $S D$. While slightly more liberal than other filters recommended

149 for similar electrode placement (e.g., Wissel \& Palaniappan, 2011, in which the authors

150 recommend a filter with cutoff frequencies of 1 and $5 \mathrm{~Hz}$ ), these parameters provided a smooth

151 baseline with little noise and clearly discernible peaks. Peaks with an amplitude at least $4 S D$

152 higher than baseline activity indicated eyeblinks. There was no difference between blink 
153 occurrences recorded manually from video, or using EOG.

154 BF data was collected for each participant in four experimental periods: two baseline

155 periods (at the beginning and end of the interview, during which the participants answered

156 personal questions), a target period (containing the mission relevant questions), and a target offset

157 period defined as the $6 \mathrm{~s}$ period following the target period (as observed by Leal \& Vrij, 2008).

158 By participant, for each experimental period, BF was quantified as the number of blinks in that

159 period divided by the mean number of blinks exhibited in the two baseline periods. This value

160 provides a measure of percent deviation in BF from baseline. Percent deviation scales the

161 frequencies to account for individual differences in BF (Leal \& Vrij, 2008). Results will be

162 described in terms of this percent deviation metric.

163 Procedure

164 The experimental protocol was an immersive mission based scenario similar to that used

165 by Leal et al. (2011) and Leal and Vrij (2008). The protocol consisted of a briefing and an

166 interview. Participants arrived at a room in an academic building and received a briefing from

167 one of the experimenters posing as a friendly agent, then went on a mock mission to deliver a

168 package to a second room in the same building. There, participants would be interviewed by

169 another experimenter role playing an anonymous agent. Participants were instructed to tell the

170 truth to the interviewer or lie based on the interviewer's response to a challenge question.

171 Correctly answering this challenge question would indicate to the participant that the interviewer

172 is friendly, and that the participant should be entirely truthful. An incorrect response to the

173 challenge question by the interviewer would indicate that the interviewer is an enemy agent to

174 whom the participant should lie about all details of the mission. Participants were randomly

175 assigned to the lying and truth telling conditions, leaving 15 truth tellers and 17 liars after

176 exclusions.

177 During the briefing in the first room, participants were informed that the agent delivering 
178 the briefing was (1) a Saint Joseph's University graduate student, (2) did not receive his/her

179 bachelor's degree from Saint Joseph's University, and (3) enjoys running. After the briefing, 180 participants were sent to deliver the package to the second room. Participants were briefed by a

181 male or female experimenter, and $t$ tests revealed no significant effect of briefer gender, all $t(30)$

$182<.31$, all $p>.75$, or participant gender, all $t(31)<1.64$, all $p>.1$, on the experimental variables.

183 Upon arrival at the second room, participants were prepared for the EOG analysis and

184 interviewed by another experimenter. Participants were interviewed through a one way mirror

185 and the voice of the interviewer was modified using a voice distortion microphone. This protocol

186 was adopted to eliminate variance attributable to the interviewer's gender and body language.

187 The interview consisted of two periods of free recall, the target period consisting of mission

188 relevant questions, and a $6 \mathrm{~s}$ period immediately following the target period (target offset period).

189 The interviewer waited $10 \mathrm{~s}$ between questioning periods.

190 During the baseline periods, participants were asked to freely recall information regarding

191 irrelevant subject matter. For one baseline period, participants were told, "Please take one minute

192 to tell me about your favorite actor or actress." Participants were asked to include in their

193 responses shows or movies in which this person has acted, this person's on screen characters, and

194 why they like this actor or actress. During the other baseline period, participants were asked to

195 describe their favorite food, and to specifically address its national origin, whether there are any

196 local restaurants in which to eat it, and whether they like it for its nutritional value or just for the

197 taste. Baseline period content was counterbalanced to eliminate order effects. Despite one

198 baseline period requiring description of a food and one of a person, there were no significant

199 differences in BF between baseline periods, indicating that each baseline recall task was similarly

200 demanding (data not shown). Likewise, both liars $(M=.59, S D=.22)$ and truth tellers $(M=.50$,

$201 S D=.13$ ) exhibited similar BF (per second) during the baseline periods, $t(30)=-1.46, p=.15$.

202 During the target period, participants answered mission relevant questions about the agent 
203 who delivered the briefing. The questions were:

$204 \quad$ 1. Who sent you?

2052 What does this person look like?

206 3. What does this person do for a living?

207 4. Did this person earn his or her bachelor's degree at SJU?

208 5. Does this person have a hobby?

209 For the three questioning periods (i.e., the two baseline periods and the target period), the 210 experimenter delivered the questions, without breaks, then allowed the participants to freely

211 recall the information and respond. In cases where participants' responses lasted less than $15 \mathrm{~s}$,

212 the experimenter prompted the participant to elaborate and continue. Participants were allowed to 213 speak for up to $120 \mathrm{~s}$. After questioning, participants completed a 7-point motivation Likert scale 214 ("How motivated were you to do well in the interview?") and were debriefed.

\section{Results}

No between groups differences were found in response length during the "favorite actor,"

217 "favorite food," or target period for liars and truth tellers, all $t(30)<.81$, all $p>.42$. Response

218 length data for each group during the experimental periods are available in Table 1. All

219 participants reported a high degree of motivation according to the Likert scale $(M=6.00, S D=$.

220 73) and condition assignment did not affect participants' reported motivation to perform well in

221 the interview, $t(29)<.001, p>.99$.

A 2 (Veracity: lying vs. truth telling) x 2 (Experimental Period: target period deviation vs.

223 target offset deviation) factorial ANOVA, with repeated measures on the second factor, was

224 conducted to assess differences in BF modulation patterns between liars and truth tellers. The

225 analysis revealed a main effect for Experimental Period, $F(1,30)=16.17, p<.001, \eta p^{2}=.35$,

226 such that participants exhibited a higher BF during the target period $(M=.98, S D=.21)$ than the

227 target offset period $(M=.71, S D=.36)$. The analysis also found an interaction effect of Veracity 
$228 \mathrm{x}$ Experimental Period, $F(1,30)=6.00, p=.020, \eta p^{2}=.167$. The Veracity $\mathrm{x}$ Experimental Period

229 interaction effect indicated that liars displayed a significantly suppressed BF during the target

230 period $(M=.85, S D=.13)$ compared to the increase exhibited by truth tellers $(M=1.12, S D=$.

$23119 ; d=1.66)$, and less BF reduction during the target offset period $(M=.74, S D=.36)$ compared

232 to truth tellers $(M=.66, S D=.34 ; d=.23$; Figure 1$)$. Followup $t$-tests indicated significant

233 differences in target period deviation for both liars, $t(16)=4.56, p<.001$, and truth-tellers, $t(14)$

$234=-2.27, p=.039$.

235

MANOVA identified between groups differences in the predictor variables, the target and

target offset deviation scores, based on veracity as the independent variable. The data satisfied the

assumption of homoscedasticity using Box's M. Hotelling’s Trace revealed a significant

238

multivariate effect of veracity on the dependent variables, $F(2,29)=9.91, p=.001, \eta p^{2}=.406$.

239 Univariate ANOVAs showed a significant effect of veracity on the target deviation score, $F(1,30)$

$240=19.95, p<.001, \eta p^{2}=.40$, but an insignificant effect on target offset deviation, $F(1,30)=.38, p$

$241=.54, \eta p^{2}=.01$. Target period deviation from baseline was therefore retained as the sole predictor

242 for discriminant analysis.

243 Discriminant analysis tested the capability of target period deviation to discriminate

244 between liars and truth tellers. The discriminant function incorporating the predictor was

245 significant, $\chi^{2}(2)=15.04, p<.001$, with $81.3 \%$ of cases correctly classified. Using the

246 discriminant function, $88.2 \%$ of liars and $73.3 \%$ of truth tellers were correctly classified,

247 indicating that the function favored sensitivity over specificity. Because of the small sample size,

248 the data were cross validated to check external validity using a jackknife procedure

249 (Lachenbruch, 1967) which is appropriate for small sample sizes (Stevens, 2009). The original

250 and cross validated classification results are shown in Table 2, and the significance and power of

251 the discriminant function are provided in Table 3. Cross validation resulted in the

252 misclassification of one truth telling participant. 


\section{Discussion}

One goal of the present study was to replicate the findings of Leal and Vrij (2008) in

255 which, during free recall, liars exhibited suppressed BF during the target period followed by a

compensatory flurry of eyeblinks, whereas truth tellers exhibited an increase in BF during the

257

258

259

260

261

262

263

264

265

266

267

268

269

270

271

272

273

274

275

276

target period. The present study found a similar difference in BF between groups during the target

period; however, neither group exhibited a compensatory flurry of eyeblinks during the target

offset period (Figure 1).

Though truth tellers' BF dynamics matched the findings of Leal and Vrij (2008), there is a

possible alternative to the anxiety explanation provided in that study that is congruent with the

cognitive demand hypothesis. Since BF reflects state cognitive demand, it is possible that the

increase from baseline observed in truth tellers during the target period is attributable to a state of

reduced cognitive demand. Recalling the recently acquired semantic information truthfully was

perhaps less demanding than retrieving autobiographical information to answer the baseline

questions. This is supported by reaction time studies on autobiographical versus semantic recall

(Haque \& Conway, 2001). Therefore, it is possible that BF changes in truth tellers are the result

of cognitive demand changes rather than anxiety or another emotional response.

The differences observed by Leal and Vrij (2008) may also be attributed to the content of

the experimental periods used in that study; during the target period, participants were given no specific instruction, while their behaviors during the baseline periods were directed. In addition, participants in the lying and truth telling conditions engaged in different behaviors; liars committed a mock crime whereas truth tellers did not. If truth tellers' actions during the target period were less complex than their directed behaviors during the baseline periods, then recalling the target period information may have been less demanding.

277 findings of Leal and Vrij (2008), who noted a flurry of compensatory blinks in liars. For this 
278

279

280

281

282

283

284

285

286

287

288

289

290

291

292

293

294

295

296

297

298

299

300

301

302

result, three proposed explanations follow. First, capturing that information may be exceptionally difficult due to the short window in which the flurry would occur. Frequency data for the target offset period is calculated over a $6 \mathrm{~s}$ period, which is significantly shorter than the other experimental periods. Second, these inconsistent results may be the result of differences in experimental protocol, such that a view of the interviewer, present in the study by Leal and Vrij (2008) but obscured in the present study, is necessary to induce these compensatory blinks. The final possibility is that the BF reduction observed in the present study is due to the fact that participants were not speaking or listening during this period. This possibility is supported by other literature on blink BF dynamics (e.g., Karson et al., 1981), which suggest that BF during silence is significantly lower than during speech or listening. These results, taken together, seem to indicate that the post-questioning compensatory flurry of blinks does not always follow deceptive responses, and depends heavily on other factors.

The second goal of the present study was to evaluate the BF modulation during a free recall test for application beyond subjective deception, to deception regarding a third party. Validating the method in this way indicates greater robustness required for application, and an advantage over GKT-based techniques. Prior research using BF (Leal \& Vrij, 2008) focused on recent subjective events, whereas the present study primarily focused on semantic details about a third party, though the target period did include a question regarding the purpose of the participant's "mission." BF characteristics were similar in the present study to those observed by Leal and Vrij (2008) for subjective deception.

While the results obtained herein indicate that it is possible to discriminate between liars and truth tellers, studies in this area do not speak to the fact that is perhaps more important in application: detecting deception within subjects. Since BF does not indicate deception, but rather cognitive demand, these techniques rely on between group comparisons to make causal inferences regarding the cause of the blink frequency suppression. Future research in this area 
303 should seek to explore BF dynamics associated with varying question content. Cognitive task

304 analysis of common interrogation questions may aid in identifying analogue questions to serve as

305 baselines (i.e., questions requiring similar cognitive demand to answer).

306 Additionally, certain ecological validity issues remain unresolved. The present study did

307 not incorporate any meaningful interval between encoding of information (briefing) and testing

308 (interview). Carmel et al. (2003) demonstrated that intervals as short as one week can

309 significantly impair accuracy of other tests of deception (specifically the GSR based GKT)

310 employed in experimental conditions. In addition, if liars are allowed to construct and rehearse an

311 alibi, this would likely reduce cognitive demand as the fabrication component of deception would

312 be removed. Therefore, the accuracy obtained in the present study should not be considered

313 externally valid.

\section{Conclusions}

316 Results of the present study suggest that a technique measuring BF reduction during

317 deception, presumably resulting from increased cognitive demand, is sufficiently robust to detect

318 deception when the suspect does not have a view of the interviewer, and when the suspect is

319 asked about a third party. Because BF data can be collected surreptitiously using webcams and

320 hidden cameras, and analyzed either in real time or post-hoc from recorded video, BF based

321 techniques warrant consideration. However, there exist a number of hurdles to application that

322 appear intrinsic to BF based techniques.

323 In the absence of between subject comparisons, it is perhaps not possible to definitively

324 attribute BF suppression to deception. To the extent that it is possible to ameliorate this

325 shortcoming, baseline content must be carefully developed and selected so as to be similarly

326 demanding as truth telling in order to detect BF reductions indicative of lying. Manipulating the

327 content of baseline and target questions could improve the sensitivity and specificity of the test. 
328 Furthermore, tests such as the GKT benefit from repetition of target questions (Ben-Shakar \&

329 Elaad, 2002), so perhaps multiple presentations of target questions, changed slightly as to require

330 the fabrication of new responses, would also increase classification accuracy. While the technique

331 offers several advantages to traditional physiological methods for lie detection, additional

332 research is required to determine if it is suitable for detecting deception within subjects.

333

334

335

336

337

338

339

340

341

342

343

344

345

346

347

348

349

350

351

352

353 Abootalebi, V., Moradi, M. H., \& Khalilzadeh, M. A. (2006). A comparison of

\section{Acknowledgements}

This work was completed to satisfy the requirements of a MS thesis. All materials and space used for this work are property of Saint Joseph's University, Philadelphia, PA. The author thanks Victoria Kurzeja for her participation as a confederate during this study. Additionally, the author thanks Ashley L. Adams, Donald S. Leitner, Philip Schatz, and Alex J. Skolnick for their input on this manuscript. 
methods for ERP assessment in a P300-based GKT. International Journal of Psychophysiology, 62, 309-320. doi:10.1016/j.ijpsycho.2006.05.009

356 Ambach, W., Bursch, S., Stark, R., \& Vaitl, D. (2010). A Concealed Information Test with multimodal measurement. International Journal of Psychophysiology, 75, 258-267.

Polygraph Testing (pp. 87-102). San Diego, CA: Academic Press.

361 Bhatt, S., Mbwana, J., Adeyemo, A., Sawyer, A., Hailu, A., \& VanMeter, J. (2009). Lying about facial recognition: An fMRI study. Brain and Cognition, 69, 382-390. doi:10.1016/j.bandc.2008.08.033

Carmel, D., Dayan, E., Naveh, A., Raveh, O., \& Ben-Shakar, G. (2003). Estimating the validity of the Guilty Knowledge Test from simulated experiments: The external validity of mock crime studies. Journal of Experimental Psychology: Applied, 9, 261-269. doi:10.1037/1076-898X.9.4.261

Conduit, R. (2012). Recording startle eye-blink in humans: Habituation, pre-pulse inhibition and conditioning [Software educational supplement]. Retrieved from http://www.adinstruments.com/downloads/contributions/Startle_Eye_Blink.pdf?

DePaulo, B. M. (1988). Nonverbal aspects of deception. Journal of Nonverbal Behavior, template=education 12, 153-162. doi: 10.1037/0022-3514.56.5.834

Drew, G. C. (1951). Variations in reflex blink-rate during visual motor tasks. Quaterly Journal of Experimental Psychology, 3, 73-88

Ekman, P. (1989). Why lies fail and what behaviors betray a lie. In J. C. Yuille (Ed.), Credibility assessment (pp. 71-82). Dordrecht: Kluwer.

380 Gronau, N., Ben-Shakar, G., \& Cohen, A. (2005). Behavioral and physiological measures 
in the detection of concealed information. Journal of Applied Psychology, 90, 147-158. doi:10.1037/0021-9010.90.1.147

383 Haque, S. \& Conway, M. A. (2001). Sampling the process of autobiographical memory construction. European Journal of Cognitive Psychology, 13, 529-547.

385

386

387
Iacono, W.G. (2007). Detection of deception. In Cacioppo, J.T., Tassinary, L.G., \& Berntson, G.G. (Eds.). Handbook of psychophysiology, 3rd ed. (pp. 688-703). New

York, NY: Cambridge University Press.

Karson, C. N., Berman, K. F., Donnelly, E. F., Mendelson, W. B., Kleinman, J. E., \& Wyatt, R. J. (1981). Speaking, thinking, and blinking. Psychiatry Research, 243-246.

Lachenbruch, P. A. (1967). An almost unbiased method of obtaining confidence intervals for the probability of misclassification in discriminant analysis. Biometrics, 23, 639-645.

Langleben, D. D. (2008). Detection of deception with fMRI: Are we there yet? Legal and Criminological Psychology, 13, 1-9. doi: 10.1348/135532507X251641

Langleben, D. D., Loughead, J. W., Bilker, W. B., Kosha, R., Childress, A. R., Busch, S. I., \& Gur, R. C. (2005). Telling truth from lie in individual subjects with fast event-related fMRI. Human Brain Mapping, 26, 262-272. doi: 10.1002/hbm.20191

Leal, S., \& Vrij, A. (2008). Blinking during and after lying. Journal of Nonverbal Behavior, 32, 187-194. doi:10.1007/s10919-008-0051-0

Leal, S., \& Vrij, A. (2010). The occurrence of eye blinks during a guilty knowledge test. Psychology, Crime, \& Law, 16, 349-357. doi: 10.1080/10683160902776843

Leal, S., Vrij, A., Mann, S. A., \& Fisher, R. P. (2011). Detecting concealed information about person recognition. Applied Cognitive Psychology, 25, 372-376. doi:10.1002/acp.1701

Lykken, D. T. (1957). A study of anxiety in the sociopathic personality. Journal of 
407 Lykken, D. T. (1959). The GSR in the detection of guilt. Journal of Applied Psychology, $408 \quad 43,385-388$. doi: $10.1037 / \mathrm{h} 0046060$

409 MacLaren, V. V. (2001). A quantitative review of the Guilty Knowledge Test. Journal of Applied $410 \quad$ Psychology, 86, 674-683.

411 Mann, S., Vrij, A., \& Bull, R. (2002). Suspects, lies, and videotape: An analysis of 412 authentic high-stake liars. Law and Human Behavior, 26, 365-376. doi: 10.1023/A:1015332606792

414 Meijer, E. H., Smulders, F. T. Y., Merckelbach, H. L. G. J., \& Wolf, A. G. (2007).

415 The P300 is sensitive to concealed face recognition. International Journal of Psychophysiology, 66, 231-237. doi:10.1016/j.ijpsycho.2007.08.001

Shah, N. J., Marshall, J. C., Zafiris, O., Schwab, A., Zilles, K., Markowitsch, H. J., \& Fink, G. R. (2001). The neural correlates of personal familiarity: A functional magnetic resonance imaging study with clinical implications. Brain, 124, 804815. doi:10.1093/brain/124.4.804

421 Seymour, T., Kerlin, J., \& Kurtz, A. (2003, August). Extending the response-time "Guilty Knowledge Test". Poster session presented at the annual meeting of the Cognitive Science Society. Amsterdam, the Netherlands.

Siegle, G. J., Ichikawa, N., \& Steinhauer, S. (2008). Blink before and after you think: Blinks occur prior to and following cognitive load indexed by pupillary responses. Psychophysiology, 45, 679-687. doi:10.1111/j.1469-8986.2008.00681.x

427 Stevens, J. P. (2009). Applied multivariate statistics for the social sciences. New York, $428 \quad$ NY: Routledge.

429 Sullivan, J. F. (2007). Gatekeeper: Memoirs of a CIA polygraph examiner. Dulles, VA: 430 Potomac Books, Inc. 
431 Verschuere, B., Crombez, G., Koster, H. W. E., Van Bockstaele, B., \& De Clerq, A.

432 (2007). Startling secrets: Startle eye blink modulation by concealed crime

433 information. Biological Psychology, 76, 52-60. doi:10.1016/j.biopsycho.2007.06.001

434 Wissel, T. \& Palaniappan, R. (2011). Considerations on strategies to improve EOG signal

435 analysis. International Journal of Artificial Life Research, 2, 6-21.

436

437

438

439 


\section{Table 1 (on next page)}

Response lengths for liars and truth tellers during experimental periods 
Experimental Group

Liars

Truth Tellers
"Favorite Food"

$M=37.76, S D=20.05$

$M=43.9, S D=27.80$
"Target"

$M=38.47, S D=14.45$

$M=33.03, S D=15.58$
"Favorite Actor"

$M=31.32, S D=14.17$

$M=32.70, S D=19.53$ 


\section{Figure 1}

Results of 2 (Veracity: Lying vs. Truth-telling) $\times 2$ (Experimental Period: Target vs.

Target Offset) ANOVA. BF for each group across experimental periods quantified as percent change from baseline.

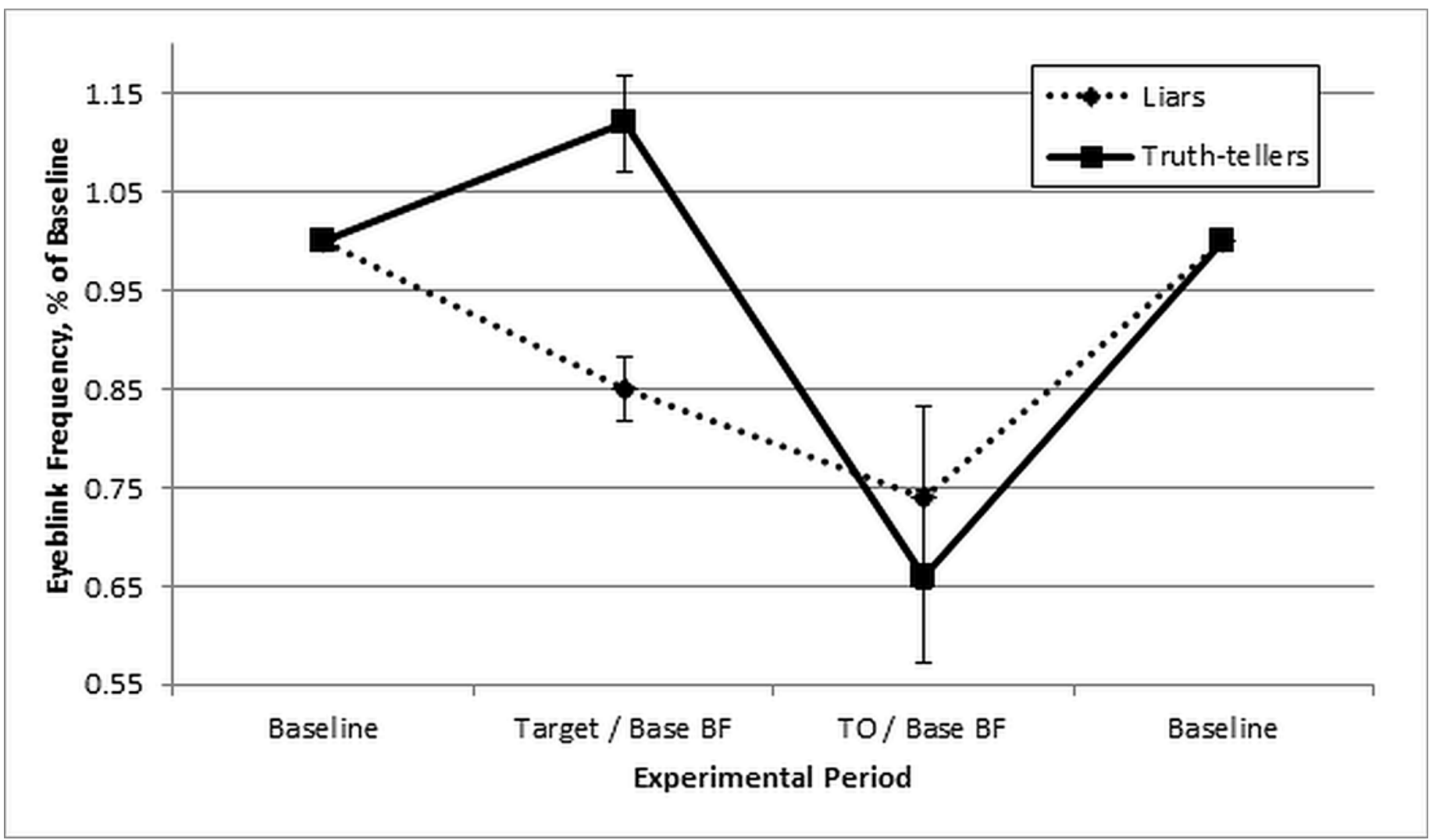




\section{Table 2 (on next page)}

Classification table for percent-change scores between experimental periods 


\begin{tabular}{|c|c|c|c|}
\hline \multirow[t]{2}{*}{ Actual } & \multicolumn{3}{|c|}{ Predicted Veracity - Original } \\
\hline & Lying & Not Lying & Total \\
\hline Lying $(N)$ & 15 & 2 & 17 \\
\hline Not Lying & 4 & 11 & 15 \\
\hline Lying (\%) & 88.2 & 11.8 & 100 \\
\hline Not Lying & 26.7 & 73.3 & 100 \\
\hline \multirow[t]{2}{*}{ Actual } & \multicolumn{3}{|c|}{ Predicted Veracity - Cross-validated } \\
\hline & Lying & Not Lying & Total \\
\hline Lying $(N)$ & 15 & 2 & 17 \\
\hline Not Lying & 5 & 10 & 15 \\
\hline Lying (\%) & 88.2 & 11.8 & 100 \\
\hline Not Lying & 33.3 & 66.7 & 100 \\
\hline
\end{tabular}

Note. $81.3 \%$ of original grouped cases correctly classified. $78.1 \%$ of cross-validated grouped cases correctly classified. 


\section{Table 3 (on next page)}

Significance of the discriminant function predicting veracity, and discriminating power of the discriminant function 
Wilk's Lambda

.601

Eigenvalue Percentage of variance

.665 d.f.

1

Significance

$<.001$ 